\title{
Combination of calcipotriol and methotrexate in nanostructured lipid carriers for topical delivery
}

\author{
This article was published in the following Dove Press journal: \\ International Journal of Nanomedicine \\ 24 February 2010 \\ Number of times this article has been viewed
}

\author{
Yin-Ku Lin ${ }^{1,2}$ \\ Zih-Rou Huang ${ }^{3}$ \\ Rou-Zi Zhuo ${ }^{3}$ \\ Jia-You Fang ${ }^{3,4}$ \\ 'Graduate Institute of Clinical \\ Medical Sciences, Chang Gung \\ University, Kweishan, Taoyuan, \\ Taiwan; ${ }^{2}$ Department of Traditional \\ Chinese Medicine, Chang Gung \\ Memorial Hospital, Keelung, Taiwan; \\ ${ }^{3}$ Pharmaceutics Laboratory, Graduate \\ Institute of Natural Products, \\ Chang Gung University, Kweishan, \\ Taoyuan, Taiwan; ${ }^{4}$ Department of \\ Pharmaceutics, College of Pharmacy, \\ King Saud University, Riyadh, \\ Saudi Arabia
}

\begin{abstract}
The combination of calcipotriol with methotrexate can strengthen the topical therapy for psoriasis. The aim of the present study was to evaluate the potential of nanostructured lipid carriers (NLCs) loaded with lipophilic calcipotriol and hydrophilic methotrexate as topical therapy. NLCs composed of Precirol ATO 5 with various amounts of squalene as the liquid lipid were prepared. The particle size, surface charge, molecular environment, drug permeation, and skin irritation of the carriers were assessed. Hyperproliferative skin was also used as a permeation barrier in this study. It was found that variations in the $\operatorname{Precirol}^{\circledR} /$ squalene ratio had profound effects on the physicochemical characteristics of the NLCs. The range of particle size of the NLC preparations was 270 to $320 \mathrm{~nm}$, with vehicles containing a higher Precirol amount exhibiting a larger diameter. NLCs with a higher Precirol/squalene ratio also showed greater polarity in their molecular environment. Calcipotriol-loaded NLC systems provided drug fluxes of 0.62 to $1.08 \mu \mathrm{g} / \mathrm{cm}^{2} / \mathrm{h}$, which were slightly higher or comparable to the $30 \%$ ethanol vehicle (control, $0.72 \mu \mathrm{g} / \mathrm{cm}^{2} / \mathrm{h}$ ). The methotrexate amount permeating the skin was 2.4 to 4.4-times greater using NLCs compared to that with the control. Dual drugloaded NLCs exhibited reduced skin permeation of calcipotriol but not methotrexate. The in vivo topical delivery examined by confocal laser scanning microscopy (CLSM) showed a good correlation with the in vitro results. These two drugs with extremely different polarities can successfully be combined in NLCs. Results suggest that NLCs may have the potential to serve as delivery carriers for antipsoriatic drugs because of enhanced drug permeation and limited skin irritation
\end{abstract}

Keywords: psoriasis, calcipotriol, methotrexate, nanostructured lipid carriers, topical delivery

\section{Introduction}

Psoriasis is one of the most common human skin diseases. It is characterized by excessive growth and aberrant differentiation of keratinocytes. ${ }^{1,2}$ Topical therapy is the mainstay of treatment for mild to moderate psoriasis and often the initial treatment for severe psoriasis. About $80 \%$ of patients with psoriasis are treated topically. ${ }^{3}$ No single topical medication is perfect by itself in the management of psoriasis; however, combined regimens with more than one medication are often beneficial. ${ }^{4}$ Calcipotriol (Figure 1) is a synthetically prepared vitamin $\mathrm{D}_{3}$ analogue used for the treatment of psoriasis. ${ }^{5}$ Calcipotriol leads to cutaneous irritant reactions in $\sim 20 \%$ of patients. ${ }^{6}$ Calcipotriol combined with other therapies may allow the use of a reduced dose, thus minimizing the skin irritation. It can be used in combination with topical corticosteroids, ultraviolet (UV)B, psoralen-UVA, acitretin, cyclosporine, tazarotene, and methotrexate. ${ }^{7-9}$
Graduate Institute of Natural Products, Chang Gung University, 259 Wen-Hwa Ist Road, Kweishan, Taoyuan 333, Taiwan Tel +88632118800 Ext 5521

Fax +88632118236

Email fajy@mail.cgu.edu.tw 


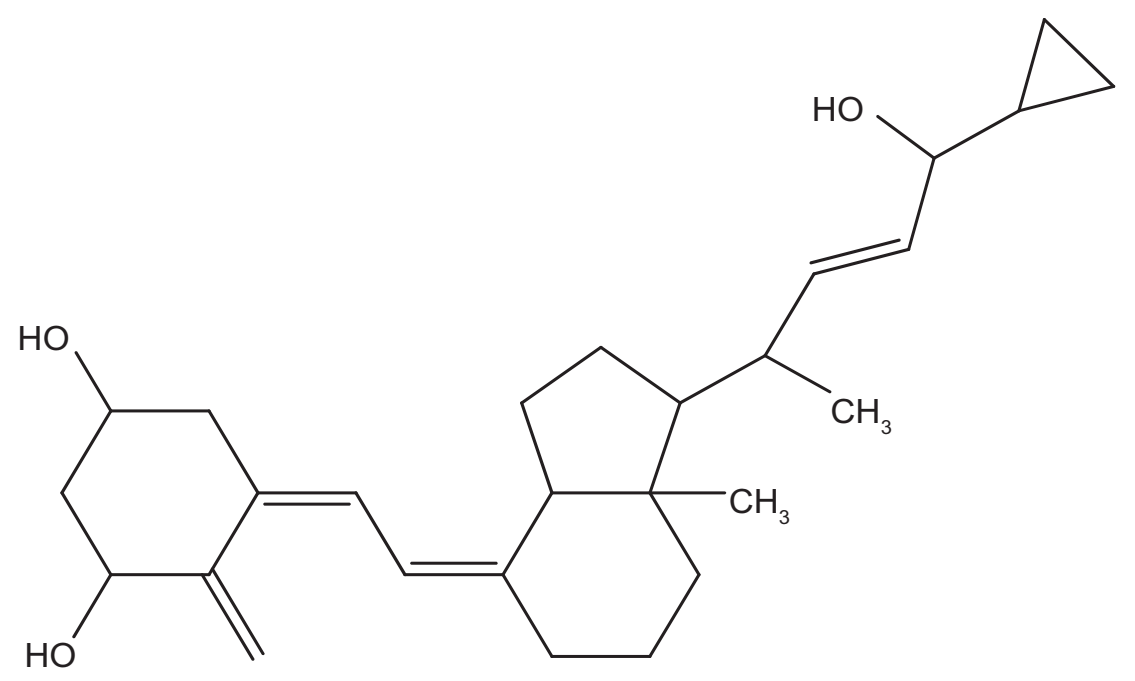

Calcipotriol

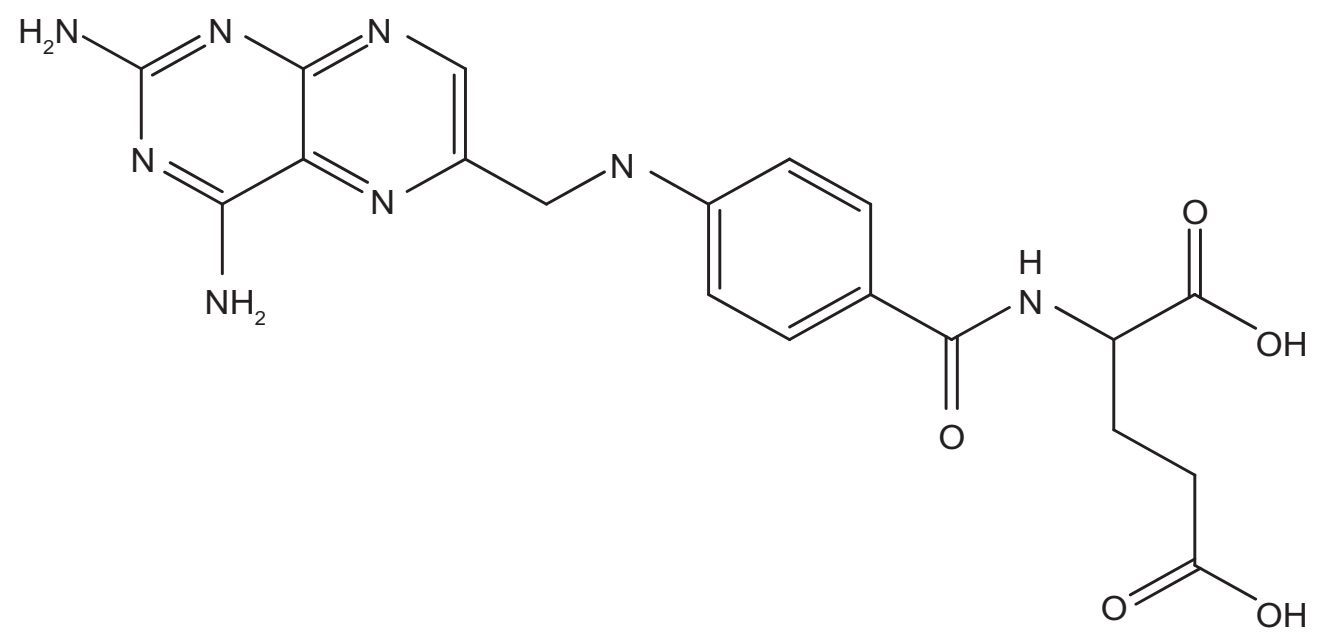

Methotrexate

Figure I Chemical structures of calcipotriol and methotrexate.

The greatest limitation to the combined therapy is the inconvenience of administration. A drug vehicle should first be applied to the skin, followed by another drug vehicle after a determined duration. The two drugs incorporated in one formulation would likely result in better patient compliance. However, it is not easy to incorporate two drugs with extremely different polarities into a single vehicle, eg, calcipotriol (partition coefficient $\log P=4.6$ ) and methotrexate (Figure 1, $\log$ $P=-2.2$ ). A carrier with both lipid and aqueous phases such as emulsions and creams may overcome this problem. Solid lipid nanoparticles (SLNs) are the new generation of oil-inwater nanoparticulate systems and are attracting attention as novel colloidal drug carriers for topical use. SLNs have many advantages, such as controlled release and enhanced bioavailability. ${ }^{10,11}$ Nanostructured lipid carriers (NLCs) are considered to be the smartest, latest generation of SLNs possessing improved properties of drug loading, modulation of the release profile, and stable drug incorporation during storage. ${ }^{12}$ NLCs are produced by mixing solid lipids with spatially incompatible lipids leading to a lipid matrix with a special structure.

At the molecular and cellular levels, calcipotriol and methotrexate have different modes of action. The combination of these two drugs can successfully be used in practice. ${ }^{13}$ Although SLNs and NLCs are used as the carriers for single drug in several studies, the study about dual drugs in one 
formulation for these nanocarriers is very few. The aim of this study was to try to incorporate calcipotriol and methotrexate in NLC systems for topical delivery. The present work evaluated the possibility of producing NLCs containing two drugs, and their delivery to the skin was examined. The physicochemical properties of the obtained NLCs, such as the size, zeta potential, and polarity, were investigated. Moreover, hyperproliferative skin was used as a skin barrier for drug permeation in order to mimic a clinical situation.

\section{Materials and methods Materials}

Calcipotriol was purchased from 21CEC (East Sussex, UK). Methotrexate, squalene, Pluronic ${ }^{\circledR}$ F68 (PF68), Nile red, sulforhodamine B (SRB), and fluorescein isothiocyanate (FITC) were supplied by Sigma Chemicals (St. Louis, MO, USA). Precirol ${ }^{\circledR}$ ATO 5, a mixture of $8 \%$ to $22 \%$ monoacyl, $40 \%$ to $60 \%$ diacyl, and $25 \%$ to $35 \%$ triacyl glyceryl palmitate and $40 \%$ to $60 \%$ stearate, was obtained from Gattefossé (Gennevilliers, France). Myverol $^{\mathrm{TM}} 18-04 \mathrm{~K}$ (palmitinic acid monoglycerides) was from Quest (Naarden, The Netherlands).

\section{Preparation of NLCs}

The Precirol and squalene mixture $(10 \%, \mathrm{w} / \mathrm{v}$ of the final product), as well as Myverol $(0.2 \%)$ were dissolved in an appropriate volume of chloroform: methanol (2:1). The organic solvent was evaporated in a rotary evaporator at $50^{\circ} \mathrm{C}$ to obtain a thin film, and solvent traces were removed by maintaining the film under a vacuum for 6 hours. The film was hydrated with PF68 (2.8\%) and double-distilled water using a high-shear homogenizer (Pro250, ProScientific, Monroe, CT, USA) for $10 \mathrm{~min}$ at $85^{\circ} \mathrm{C}$. The mixture was further treated using a probe-type sonicator (VCX600, Sonics and Materials, Newtown, CT, USA) for $30 \mathrm{~min}$ at $85^{\circ} \mathrm{C}$. The total volume of the final product was $10 \mathrm{~mL}$. Calcipotriol $(0.06 \%)$ and methotrexate $(0.08 \%)$ were respectively positioned in the lipid and aqueous phases.

\section{Determination of the particle size and zeta potential}

The mean particle size (z-average) and zeta potential of the NLCs were measured by photon correlation spectroscopy (Nanosizer ZS90, Malvern, Worcestershire, UK) using a He$\mathrm{Ne}$ laser at a wavelength of $633 \mathrm{~nm}$. Photon correlations of spectroscopic measurements were carried out at a scattering angle of $90^{\circ}$. The NLCs were diluted 1:100 with water before the measurement.

\section{Molecular environment of NLCs}

The lipophilic fluorescent marker, Nile red, was used as the model solute, and the molecular environment (polarity) was determined by fluorometric spectrophotometry based on the solvatochromism of Nile red. The NLCs with 1 ppm Nile red were prepared as described above. Emission fluorescence spectra were determined with a Hitachi F-2500 spectrometer (Tokyo, Japan). The spectra of NLCs with Nile red were recorded with both slit widths set to $10 \mathrm{~nm}$. The $\lambda_{\text {ex }}$ was fixed at $546 \mathrm{~nm}$, and the emission spectra were recorded from 570 to $700 \mathrm{~nm}$ at a scanning speed of $300 \mathrm{~nm} / \mathrm{min}$.

\section{Partition coefficient $(\log P)$ of drugs between the lipid and water phases}

Partition coefficients were determined by equilibrating the drug partitioning between a melted lipid phase and a water phase. Three milligrams of calcipotriol (or methotrexate) was dispersed in a mixture of squalene (or melted Precirol) (1 g) and $1 \mathrm{ml}$ of water and shaken for $10 \mathrm{~min}$ in a water bath at $85^{\circ} \mathrm{C}$. The aqueous phase was separated by centrifugation at $5500 \mathrm{rpm}$ for $10 \mathrm{~min}$. The aqueous phase was subsequently filtered through a PVDF membrane with a pore size of $0.45 \mu \mathrm{m}$. The drug concentration in the water phase was determined by HPLC.

\section{High-performance liquid chromatographic (HPLC) analysis of the drugs}

The HPLC system included a Hitachi L-2130 pump, a Hitachi L-2200 sample processor, and a Hitachi L-2400 UV detector. A 25-cm-long, 4-mm inner diameter stainless steel C18 column (Merck, Darmstadt, Germany) was used. The mobile phases consisted of methanol: water $(80: 20)$ for calcipotriol and acetonitrile:water (15:85) at $\mathrm{pH} 2.7$ adjusted with phosphoric acid for methotrexate. The flow rate was $1 \mathrm{ml} / \mathrm{min}$. The UV detector was set to wavelengths of 265 and $303 \mathrm{~nm}$ for calcipotriol and methotrexate, respectively.

\section{In vitro skin permeation}

The skin permeation of the antipsoriatic drugs was measured using a Franz cell assembly. Full-thickness dorsal skin of female nude mice ( 8 weeks old) was mounted between the donor and acceptor compartments. A cellulose membrane (Cellu-Sep $^{\circledR}$ T2, with a molecular weight cutoff of 6000 to 8000) was also used as a barrier in the experiments for examining drug release from the formulations. The donor medium consisted of $0.5 \mathrm{~mL}$ of vehicle containing drugs. 
The actual amount of calcipotriol and methotrexate in donor was $300 \mu \mathrm{g}$ and $400 \mu \mathrm{g}$, respectively. The receptor medium (5.5 mL) was ethanol: $\mathrm{pH} 7.4$ buffer (3:7) to maintain sink conditions. The available diffusion area between cells was $0.785 \mathrm{~cm}^{2}$. The stirring rate and temperature of receptor were respectively kept at $600 \mathrm{rpm}$ and $37^{\circ} \mathrm{C}$. The temperature of skin surface could be maintained at about $32^{\circ} \mathrm{C}$ after this setting, which was near the in vivo status. At appropriate intervals, $300-\mu \mathrm{L}$ aliquots of the receptor medium were withdrawn and immediately replaced with equal volumes of fresh buffer. The cumulative amounts of both drugs were determined by HPLC.

\section{Induction of hyperproliferative skin for in vitro skin permeation}

Epidermal hyperproliferation simulating psoriasis-affected skin was achieved by a tape-stripping technique. ${ }^{14}$ Female nude mice ( 8 weeks old) were used in this study. All animal experiments were reviewed and approved by the Institutional Animal Care Committee at Chang Gung University (approval number: CGU07-09). Animals were housed and handled according to institutional guidelines. The dorsal skin of a mouse was stripped using cellophane tape $\left(3 \mathrm{M} \mathrm{Scotch}{ }^{\circledR}\right)$ twice daily for 5 days. The stripping was repeated 10 times for each process. After 5 days, the skin was monitored by examining the transepidermal water loss (TEWL) with an evaporimeter (TM300, Courage and Khazaka, Köln, Germany). The skin was excised until the TEWL values reached 8 to $10 \mathrm{~g} / \mathrm{m}^{2} / \mathrm{h}$. The sample number of this experiment was four.

\section{In vivo SRB and FITC delivery examined by Confocal Laser Scanning Microscopy (CLSM)}

Localization of two dyes, SRB and FITC, within nude mouse skin was determined by CLSM after in vivo topical application. A glass cylinder with an available area of $0.785 \mathrm{~cm}^{2}$ was attached to the back skin of a nude mouse with glue. SRB and/or FITC in the vehicles at a concentration of $0.01 \mathrm{mM}$ was added to each cylinder. The application duration was 2 hours. After excising the skin, it was wiped with a cotton cloth. The depth of penetration of SRB and FITC was directly examined by CLSM. The skin thickness was optically scanned at different increments through the z-axis of a Leica TCS SP2 confocal microscope (Wetzlar, Germany). Optical excitation was carried out with a 543-nm He-Ne laser, and $\lambda_{\text {em }}$ was detected at 560 to $620 \mathrm{~nm}$ for SRB.
The $\lambda_{\text {ex }}$ for FITC was $488 \mathrm{~nm}$ from an Ar laser, and the $\lambda_{\text {em }}$ was detected at 496 to $559 \mathrm{~nm}$.

\section{In vivo skin irritation test}

A $0.6-\mathrm{mL}$ aliquot of NLCs without drugs was spread uniformly over a sheet of non-woven polyethylene cloth $(1.5 \times 1.5 \mathrm{~cm})$, which was then applied to the back area of a nude mouse. The polyethylene cloth was fixed with Tegaderm $^{\circledR}$ adhesive dressing (3M, USA) and Fixomull ${ }^{\circledR}$ stretch adhesive tape (Beiersdorf AG, Germany). After 24 hours, the cloth was removed, and the treated skin area was swabbed clean with a cotton wool swab. After withdrawal of the vehicle for $30 \mathrm{~min}$, TEWL, colorimetric parameters, and the $\mathrm{pH}$ of the applied skin were measured. TEWL was recorded using a Tewameter ${ }^{\circledR}$ (TM300, Courage and Khazaka, Köln, Germany). The TEWL was automatically calculated and expressed in $\mathrm{g} / \mathrm{m}^{2} / \mathrm{h}$. A spectrocolorimeter (CD100, Yokogawa Electrical, Tokyo, Japan) was used to measure the skin erythema $\left(\mathrm{a}^{*}\right)$. The instrument recorded color reflectance three-dimensionally $\left(\mathrm{L}^{*}, \mathrm{a}^{*}\right.$, and $\left.\mathrm{b}^{*}\right)$ as recommended by the Commission Internationale de l'Eclairage (CIE). The skin surface $\mathrm{pH}$ was determined by Skin-pH-Meter ${ }^{\circledR} \mathrm{PH}$ 905 (Courage and Khazaka). An adjacent untreated site was used as a baseline standard for each determination. The temperature and relative humidity in the laboratory were kept at $26^{\circ} \mathrm{C}$ and $55 \%$, respectively. The sample number for each experiment was six $(n=6)$.

\section{Statistical analysis}

Statistical analysis of differences between different treatments was performed using unpaired Student's $t$-test. A 0.05 level of probability was taken as the level of significance.

\section{Results}

\section{Physicochemical characterization}

NLCs made of Precirol and squalene as core materials were stabilized with Myverol and PF68. NLC dispersions composed of a 10\% lipid phase were prepared using different ratios of Precirol and squalene, ie, 3:7 (NLC-3P), 5:5 (NLC-5P), and 7:3 (NLC-7P). First, the partition coefficients between the lipid and water was determined experimentally. As shown in Table 1, a greater amount of calcipotriol was incorporated in the lipid phase compared to methotrexate. Calcipotriol can partition into Precirol easier than into squalene $(P<0.05)$. The same trend was observed for methotrexate although the discrepancy between the two lipids was limited. 
Table I The partition coefficient $(\log P)$ between lipids and water of calcipotriol and methotrexate

\begin{tabular}{lll}
\hline Lipid & Calcipotriol & Methotrexate \\
\hline Precirol & $3.86 \pm 0.11$ & $-0.70 \pm 0.07$ \\
Squalene & $2.05 \pm 0.05$ & $-0.91 \pm 0.02$ \\
\hline
\end{tabular}

Note: Each value represents the mean \pm SD $(n=5)$.

Average diameters, polydispersity indexes (PDI), and surface charges of the developed NLCs are listed in Table 2. The composition of the nanoparticle core significantly affected the size $(P<0.05)$. The results showed that when increasing the Precirol concentration from $3 \%$ to $7 \%$, the mean particle size increased from 267 to $320 \mathrm{~nm}$. PDI were generally below 0.3 for all formulations, with no significant difference $(P>0.05)$ among the systems with various lipid ratios. Zeta potentials of the NLCs were -42 to $-45 \mathrm{mV}$. A negligible effect of the lipid core on the surface charge was observed. NLC-7P showed a slightly lower negative charge $(P<0.05)$ compared to the other systems.

The absorption bands of Nile red vary in shape, position, and intensity with the nature of the environment. The emission maximum of Nile red in organic solvents is near $600 \mathrm{~nm}$. The emission spectra of Nile red in the nanoparticulate systems are shown in Figure 2. When incorporated into the NLCs, the increased re-orientation probability of the surrounding water molecules led to emission shifts to longer wavelengths. The fluorescence is quenched in a more-hydrophilic environment. The results indicated an increasing trend of hydrophilicity of NLC-3P $>$ NLC-5P $\approx$ NLC-7P. The fluorescence intensities at $600 \mathrm{~nm}$ of NLC-5P and NLC-7P were comparable.

\section{In vitro skin permeation}

A key issue investigated in this study was the feasibility of using NLCs for the delivery of a double drug combination. The permeation abilities of calcipotriol and methotrexate were evaluated in vitro using Franz diffusion cells. Figure 3A shows the representative permeation kinetics of calcipotriol

Table 2 The characterization of the nanostructured lipid carriers (NLCs) by particle size, polydispersity index (PDI), and zeta potential

\begin{tabular}{llll}
\hline Formulation & Size $(\mathbf{n m})$ & PDI & Zeta potential $(\mathbf{m V})$ \\
\hline NLC-3Pa & $267.3 \pm 12.3$ & $0.25 \pm 0.04$ & $-44.6 \pm 1.4$ \\
NLC-5P & $304.5 \pm 19.0$ & $0.31 \pm 0.13$ & $-45.2 \pm 1.1$ \\
NLC-7P & $319.5 \pm 12.2$ & $0.29 \pm 0.08$ & $-41.8 \pm 0.9$ \\
\hline
\end{tabular}

aNLC-3P, the inner phase composed of Precirol:squalene $=3: 7$.

${ }^{b} \mathrm{NLC}-5 \mathrm{P}$, the inner phase composed of Precirol:squalene $=5: 5$.

CNLC-7P, the inner phase composed of Precirol:squalene $=7: 3$.

Note: Each value represents the mean \pm SD $(n=3)$. from NLCs in the absence of methotrexate. The control vehicle was $30 \%$ ethanol in water. The cumulative amounts of calcipotriol at different times in the receptor are shown, and the fluxes $\left(\mu \mathrm{g} / \mathrm{cm}^{2} / \mathrm{h}\right)$ are summarized in Figure. 4A. For the control group, the flux of calcipotriol was $0.72 \mu \mathrm{g} / \mathrm{cm}^{2} / \mathrm{h}$. NLC-5P $\left(1.08 \mu \mathrm{g} / \mathrm{cm}^{2} / \mathrm{h}\right)$ and NLC-7P $\left(1.01 \mu \mathrm{g} / \mathrm{cm}^{2} / \mathrm{h}\right)$ clearly promoted calcipotriol penetration compared to the control $(P<0.05)$. NLC-3P $\left(0.62 \mu \mathrm{g} / \mathrm{cm}^{2} / \mathrm{h}\right)$ showed a comparable flux $(P>0.05)$ to the $30 \%$ ethanol vehicle. Figure $4 \mathrm{~A}$ also compares calcipotriol permeation of a single drug with those permeated from NLCs loaded with dual drugs. All calcipotriol fluxes were reduced after incorporation of methotrexate in the systems, with the control and NLC-5P showing significant reductions $(P<0.05)$.

When a drug is applied to the skin, two consecutive physical events may become rate-limiting steps in cutaneous permeation: the release of the drug from the vehicle and its penetration through the skin barrier. Hence the ability of NLCs to deliver calcipotriol was examined by determining the release rate of the drug across cellulose membranes as depicted in Figure 4B. There was no significant difference $(P>0.05)$ among the release rates from the control, NLC-5P, and NLC-7P. NLC-3P exhibited a higher rate $(P<0.05)$ than the other vehicles tested. Similar to the flux profiles, the addition of methotrexate in the formulations decreased calcipotriol release.

The cumulative amount-time profiles of methotrexate permeation are given in Figure. 3B. Figure 5A shows the calculated methotrexate flux from $30 \%$ ethanol and NLCs. The control vehicle provided a flux of $0.25 \mu \mathrm{g} / \mathrm{cm}^{2} / \mathrm{h}$ for methotrexate delivery. Compared to the control, enhanced permeation was achieved with the NLCs. Methotrexate loading in the NLCs significantly increased the transport by 4.4-, 2.4-, and 3.0-fold, to $1.11,0.61$, and $0.75 \mu \mathrm{g} / \mathrm{cm}^{2} / \mathrm{h}$, respectively, for NLC-3P, NLC-5P, and NLC-7P. There were no significant differences $(P>0.05)$ in the release rates of methotrexate from all tested formulations (Figure 5B). Neither the flux nor release rate changed after adding calcipotriol to methotrexate-loaded vehicles $(P>0.05)$.

\section{In vitro skin permeation via hyperproliferative skin}

Calcipotriol and methotrexate are extensively used for treating hyperproliferative skin diseases. Table 3 compares the fluxes across normal and hyperproliferative skin. NLC-5P with dual drugs was used as the representative model NLC preparation in this experiment. The $30 \%$ ethanol medium and NLC-5P showed no significant differences $(P>0.05)$ in calcipotriol 
Table 3 Comparison of calcipotriol and methotrexate flux $\left(\mu \mathrm{g} / \mathrm{cm}^{2} / \mathrm{h}\right)$ between normal and hyperproliferative skins from the delivery carriers with dual drugs

\begin{tabular}{llllll}
\hline Formulation & \multicolumn{2}{l}{ Calcipotriol } & & \multicolumn{2}{l}{ Methotrexate } \\
\cline { 2 - 3 } \cline { 5 - 6 } & Normal skin & Hyperproliferative skin & & Normal skin & Hyperproliferative skin \\
\hline $30 \%$ Ethanol & $0.52 \pm 0.03$ & $0.50 \pm 0.09$ & $0.28 \pm 0.07$ & $0.42 \pm 0.10^{*}$ \\
NLC-5 $\mathrm{P}^{\mathrm{a}}$ & $0.66 \pm 0.17$ & $0.55 \pm 0.06$ & $0.64 \pm 0.02$ & $1.14 \pm 0.18^{*}$ \\
\hline
\end{tabular}

${ }^{a}$ NLC-5P, the inner phase composed of Precirol:squalene $=5: 5$.

$* P<0.05$ as compared to the value of normal skin.

Note: Each value represents the mean $\pm S D(n=4)$.

permeation between the two skin types. Hyperproliferative skin exhibited higher methotrexate permeation $(P<0.05)$ compared to normal skin; in particular, NLC-5P showed the greatest difference.

\section{In vivo SRB and FITC delivery examined by CLSM}

More in vivo studies should be conducted to support the in vitro findings. In order to explore the correlative information about the skin targeting of NLCs, distributions of SRB and FITC in the skin were investigated using CLSM. The hydrophilic FITC likely resided in the external phase. SRB is more lipophilic than FITC. ${ }^{15}$ It is believed that SRB may appear in the inner phase of NLCs. Figure 6 illustrates the results obtained from CLSM following topical delivery of SRB loaded in 30\% ethanol (Figure 6A) and NLC-5P (Figure 6B) without FITC incorporation. The skin thickness was optically scanned at $\sim 10-\mu \mathrm{m}$ increments for 12 fragments from the surface of the skin (left to right, top to bottom). No or a negligible fluorescence response from the skin was observed for the $30 \%$ ethanol vehicle. It can be seen that the intensity of red fluorescence was much greater from NLCs than from the control. The fluorescence in the horizontal section at 30 to $75 \mu \mathrm{m}$ was very strong and showed that SRB was rich in the epidermis and upper dermis. Then the intensity gradually decreased with increasing depth. The addition of FITC to the formulations did not significantly alter the fluorescence signal of SRB (Figures 6C, D). This result somewhat differs from that of the in vitro experiment.

The horizontal sectioning revealed green fluorescence for FITC as shown in Figure 7. There was almost no signal in skin treated with the FITC-loaded 30\% vehicle (Figure 7A). Figure 7B depicts an increase in the fluorescence intensity with application of FITC-loaded NLCs. The amounts of FITC in viable epidermis and the superficial dermis were higher following the application of NLCs. The green signal of the systems with dual drugs was similar to that with FITC alone (Figures 7C, D).

\section{In vivo skin irritation test}

To ensure that the topical preparations are innocuous, it is necessary to study irritation caused by NLCs by determining their toxicity. TEWL, $\mathrm{pH}$, and erythema ( $\left.\mathrm{a}^{*}\right)$ were used to evaluate the preliminary safety of NLC-5P in vivo. The $\Delta$ value (the value of the treated site minus the value of an

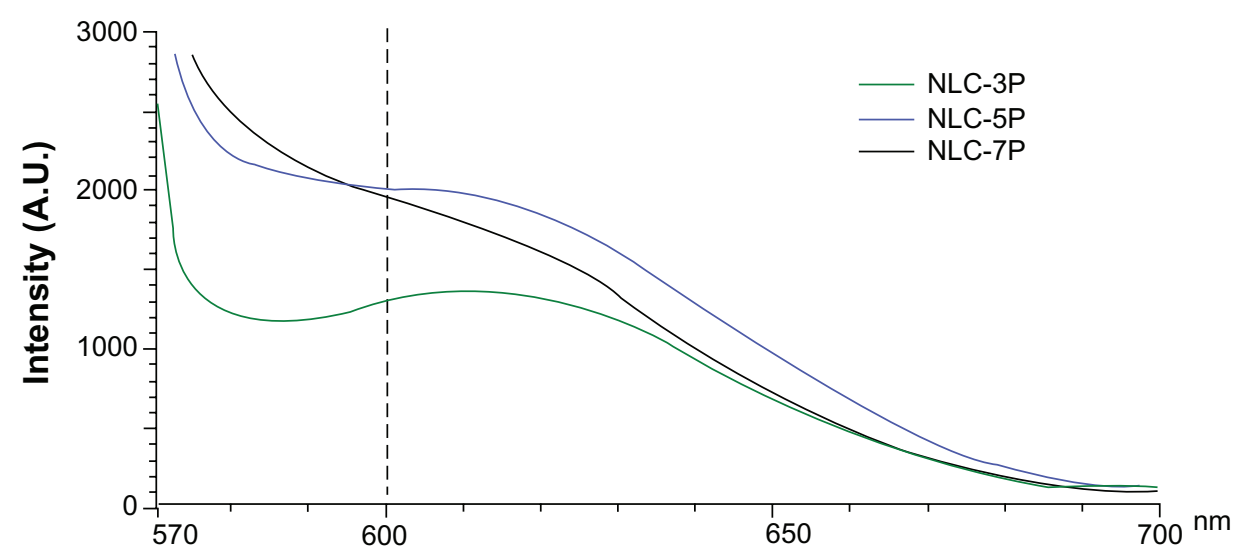

Figure 2 Fluorescence emission spectra of Nile red (I pPm) in nanostructured lipid carrier (NLC) systems with different Precirol/squalene ratios. Note: A.U. on the $\mathrm{x}$-axis means arbitrary unit. 
A

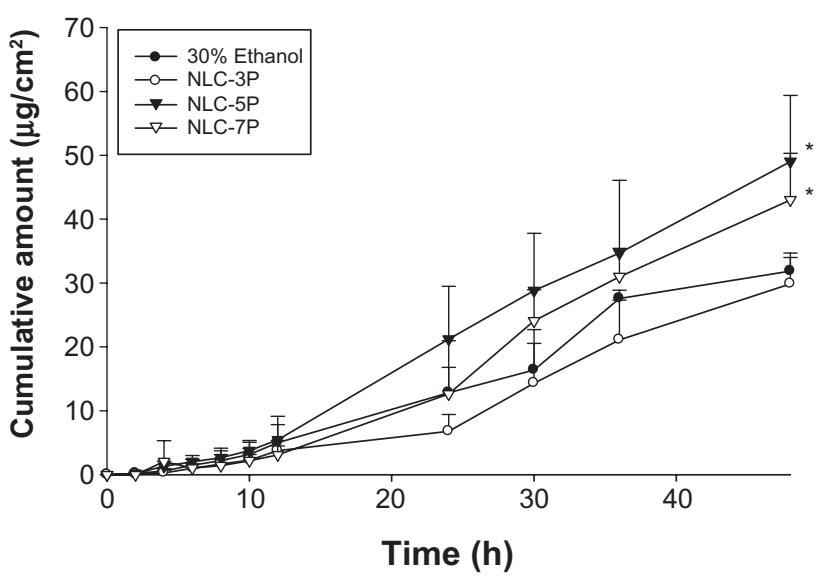

B

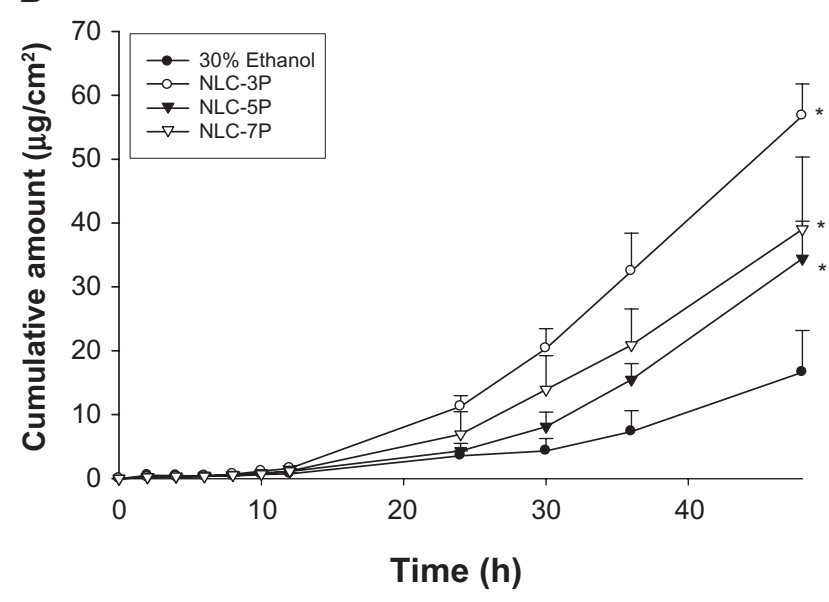

Figure 3 In vitro cumulative amount $\left(\mu \mathrm{g} / \mathrm{cm}^{2}\right)$-time profiles of calcipotriol $(\mathbf{A})$ and methotrexate (B) from 30\% ethanol (control) and nanostructured lipid carrier (NLC) systems with different Precirol/squalene ratios across nude mouse skin.

Note: Each value represents the mean $\pm \operatorname{SD}(n=4)$.

adjacent untreated site) was determined after a 24-hour administration as shown in Figure 8. No significant skin irritation was noted when the bar of the standard deviation (SD) passes across the zero line in Figure 8. Although these values increased after application of the NLCs, no significant irritation was detected. This suggests tolerable changes in the skin with the topical application of NLCs.

\section{Discussion}

Larger particles were seen in NLCs with low squalene loads. It is expected that the crystal order in the inner core is greatly disrupted by incorporating a liquid lipid although the carrier remains solid. ${ }^{16}$ The addition of a liquid lipid tends to promote the formulation of a small particle population as result of the higher molecular mobility of the matrix. ${ }^{17,18}$ PF68 is a non-ionic species. The negative surface charge shown by NLCs is believed to have resulted in the ionization of Myverol. Some free fatty acids derived from the hydrolysis of monoglycerides in Myverol may have occurred, contributing to the negative charge at the interface. The zeta potentials of NLC-3P and NLC-5P indicated that additional negative charges existed compared to NLC-7P. This means that more Myverol may be exposed at the surface. Myverol is a lipophilic emulsifier. According to the results of examining the molecular environment, the lipophilicity of the particles decreased with an increase in the amount of squalene. Myverol molecules tend to escape the inner core with its low lipophilicity, thus exposing themselves in the shell of the particles. All produced NLCs possessed high zeta potentials for electrostatic repulsion, suggesting good physical stability. ${ }^{19,20}$

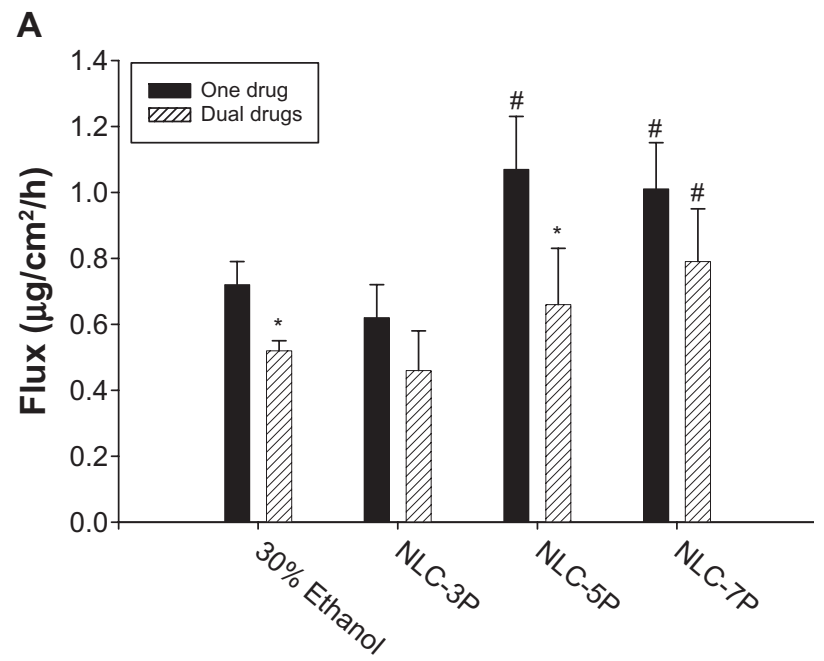

B

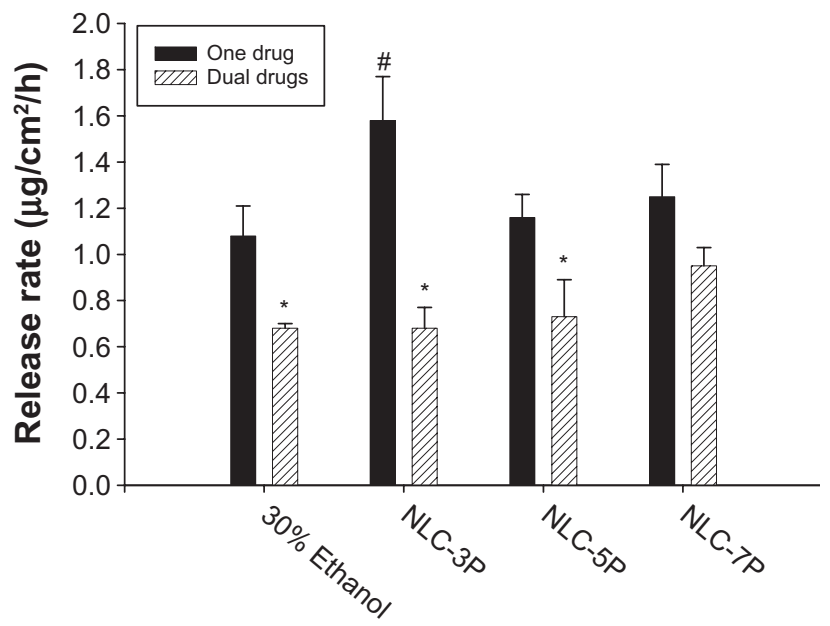

Figure 4 Flux (A) and release rate (B) of calcipotriol from 30\% ethanol (control) and nanostructured lipid carrier (NLC) systems with different precirol/squalene ratios with or without methotrexate loading.

Notes: Each value represents the mean \pm SD $(n=4)$; \# compared with $30 \%$ ethanol group; *compared with the system with one drug. 


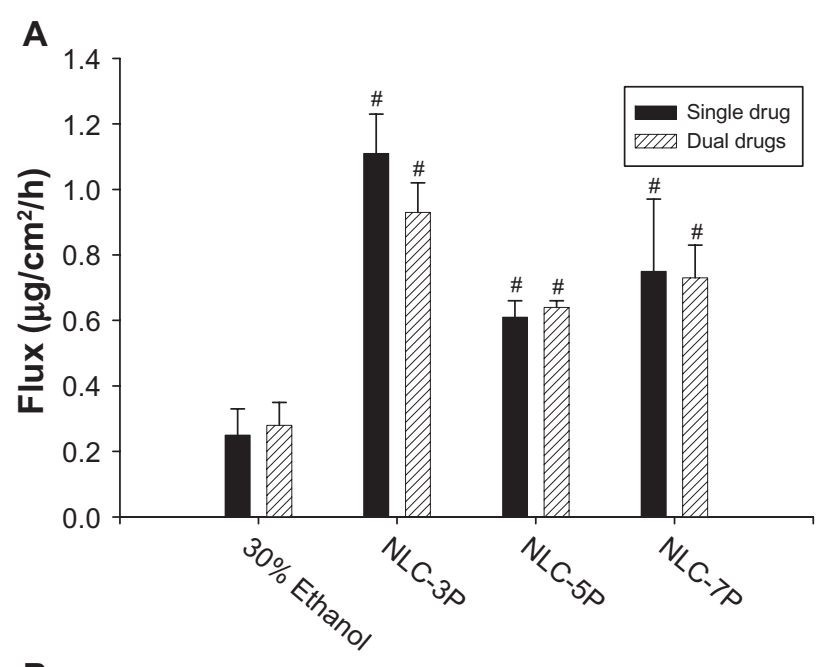

B

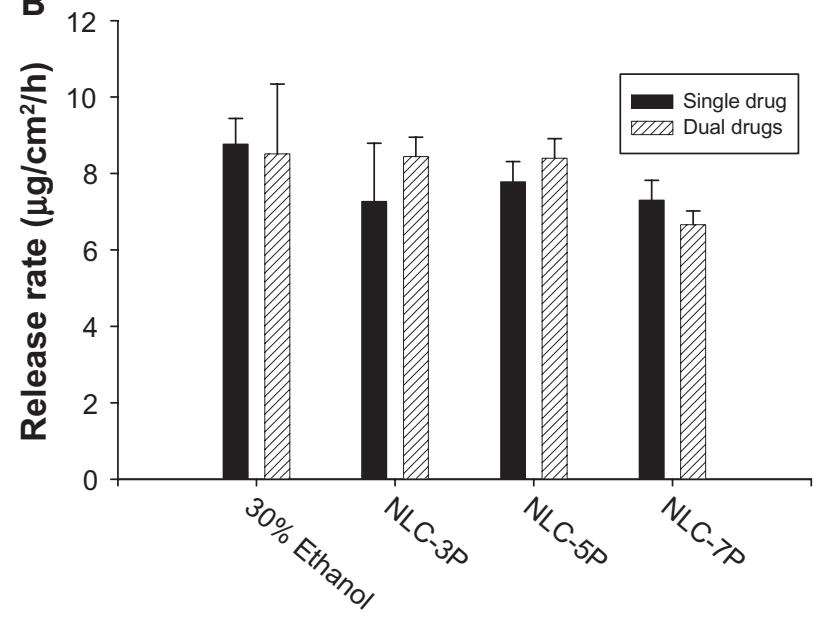

Figure 5 Flux (A) and release rate (B) of methotrexate from 30\% ethanol (control) and nanostructured lipid carrier (NLC) systems with different Precirol/squalene ratios with or without calcipotriol loading.

Notes: Each value represents the mean $\pm S D(n=4)$; \# compared with $30 \%$ ethanol group; *compared with the system with one drug.

The molecular environment was elucidated be examining the fluorescence intensity due to the solvatochromism of Nile red, a lipophilic benzophenoxazone dye. The fluorescence is quenched in aqueous media. ${ }^{21}$ Increasing Precirol in the NLCs generally created a more-lipophilic compartment for Nile red. The pronounced lipophilic character of Precirol expressed by a low HLB of 2 may have contributed to this result. Since Nile red shows only a weak intensity in a polar environment, the increase in the intensity with increasing Precirol indicates that the dye was transferred from the aqueous media or the interface to the less-polar inner core..$^{22}$ That is, Nile red in NLCs was preferentially found in the crystalline lipid phase. Hence it can be inferred that the lipophilic calcipotriol was largely embedded in the inner phase of the NLCs with the higher Precirol ratio. This inference can be confirmed by the greater partitioning of calcipotriol to Precirol than to squalene.
The drug permeating across the skin in the in vitro status dictates the amount available for skin absorption for some antipsoriatic drugs such as psoralens and methotrexate. ${ }^{23,24}$ Hence, drug flux may be an indicator of the drug absorption to the targeted skin tissue. The kinetics of drug permeation are helpful to elucidate the mechanisms of drug aborption via the skin. Release of drug from the vehicle must occur before the drug can permeate into the skin. It was found that more calcipotriol was released from the system with less Precirol (NLC-3P) compared to the others. The encapsulated drug in particles diffuses to the surface of the inner phase and undergoes partitioning between the lipid and aqueous phases. ${ }^{25}$ The drug amount in the outer shell and on the particle surface is released in the form of a burst. ${ }^{26}$ According to the results of the environmental polarity of the NLCs, calcipotriol may have largely been embedded in the interface of NLC-3P. The $\log P$ profiles also showed that calcipotriol much more easily was partitioned from squalene to the aqueous phase than that partitioned from Precirol. The expelled calcipotriol is poorly soluble in water, and hence the thermodynamic activity of calcipotriol increases. The progression of thermodynamic activity can explain the high drug diffusion of NLC-3P. ${ }^{27}$ The Precirol/squalene ratio can alter the structure of the particles, hence influencing drug release. The addition of a liquid lipid into the solid lipid matrix caused the crystal order of the NLCs to become more imperfect, and the loaded drug was more easily released. ${ }^{28,29}$ Another factor contributing to the rapid release of NLC-3P was its smaller particle size. The small size should create a larger total surface area, and an increased release rate would be expected. ${ }^{27}$

It can be seen that the release rate of calcipotriol is close to the flux value for all carriers examined. This indicates that calcipotriol easily penetrated into the stratum corneum (SC) from the vehicle. The relationship between the release rate and flux of the four vehicles tested was low (partition coefficient $r^{2}=0.263$ ). This suggests that partitioning into the skin and subsequent penetration are predominant steps in topical calcipotriol delivery. Contrary to the results of release, NLC-5P and NLC-7P provided higher drug permeation rates than did NLC-3P. NLCs with a higher Precirol/squalene ratio even had enhanced calcipotriol flux compared to the control. The mechanisms through which this occurred are not clear, and different hypotheses have been put forth. These include enhancement of solubility, the large surface area due to the nanoparticulate size, an occlusive effect, and a penetration enhancer effect. . $^{30,31}$

In general, intact particles are considered to be unable to permeate the SC. ${ }^{18,32}$ Lipid nanoparticles can deliver 


\section{A}

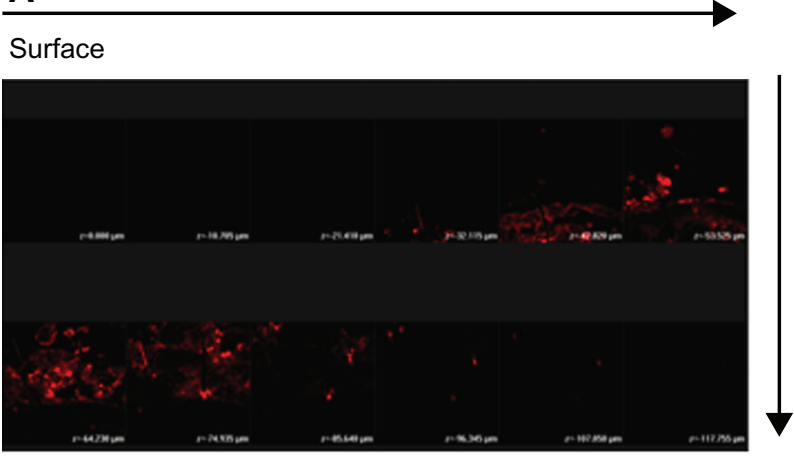

Bottom

\section{C}

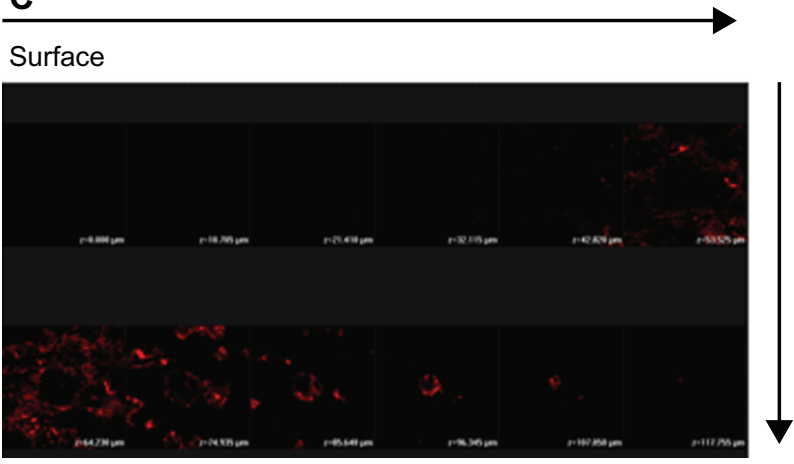

Bottom
B

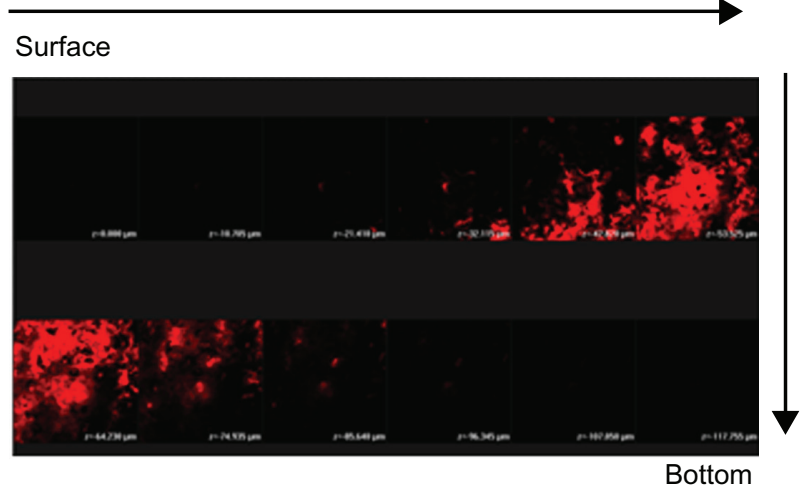

D

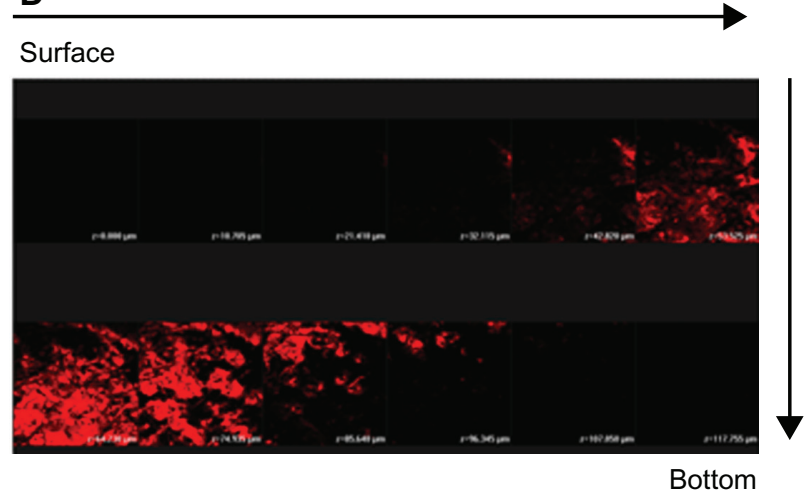

Figure 6 Confocal laser scanning microscopic (CLSM) micrographs of nude mouse skin after the in vivo topical administration of sulforhodamine B (SRB) via the skin for 2 hours from $30 \%$ ethanol (control) without fluorescein isothiocyanate (FITC) loading (A) NLC-5P without FITC loading (B) $30 \%$ ethanol (control) with FITC loading (C) NLC-5P with FITC loading (D).

Notes: The skin specimen was viewed by CLSM at $\sim 10-\mu \mathrm{m}$ increments through the Z-axis from skin surface. The arrows indicate the skin fragments from surface to bottom.

substances by interactions of the lipids used to construct the particles, and skin surface lipids.,22,32 Precirol showed a higher lipophilicity than squalene, and thus might have a greater affinity for the SC. Larger amounts of NLC particles with higher Precirol loadings were able to partition into the SC. Calcipotriol might be transported with the lipid. A partitioning of NLC-5P and NLC-7P into the SC would result in a high accumulation of the drug. An increase in the concentration gradient leads to increased diffusion pressure of the drug into the skin. ${ }^{31,33}$ CLSM results also indicated that much greater amounts of the lipophilic SRB penetrated into the skin from NLCs than from the $30 \%$ ethanol vehicle.

Increased adhesiveness to surfaces is a general property of ultra-fine materials. Lipid nanoparticles adhering to the skin form a film which subsequently leads to an occlusive effect. ${ }^{12,26}$ The amount of encapsulated drug reaching the site of action will increase. This effect should be meaningful for calcipotriol since the therapeutic response to calcipotriol can be increased by occlusion with a polyethylene film or hydrocolloid dressing. ${ }^{34,35}$ The use of NLCs should be a reliable basis for enhancing the capability of topical drug delivery due to their construction. A previous study proved that fatty acids can be skin penetration enhancers of calcipotriol. ${ }^{6}$ The free fatty acids derived from Precirol and Myverol in the particles may play this role of promoting calcipotriol permeation. This might explain the higher flux from NLC systems with a higher Precirol concentration.

Most of the formulations showed reduced calcipotriol release/permeation after methotrexate incorporation, although the differences were not always statistically significant. The incorporation of dual drugs into NLCs did not significantly alter the particle size or zeta potential $(P>0.05$, data not shown). This may have been due to the extremely low dose of the drugs. The physicochemical properties of NLCs cannot explain this reduction. The possibility of complex formation between calcipotriol and methotrexate molecules was considered. The $-\mathrm{OH}$ groups in the cyclohexane moiety of calcipotriol may interact with $-\mathrm{NH}_{2}$ groups in methotrexate's structure to form $\mathrm{H}$-bonding. Moreover, the electronegative oxygen in the $-\mathrm{COOH}$ and $-\mathrm{CONH}$ groups of methotrexate 

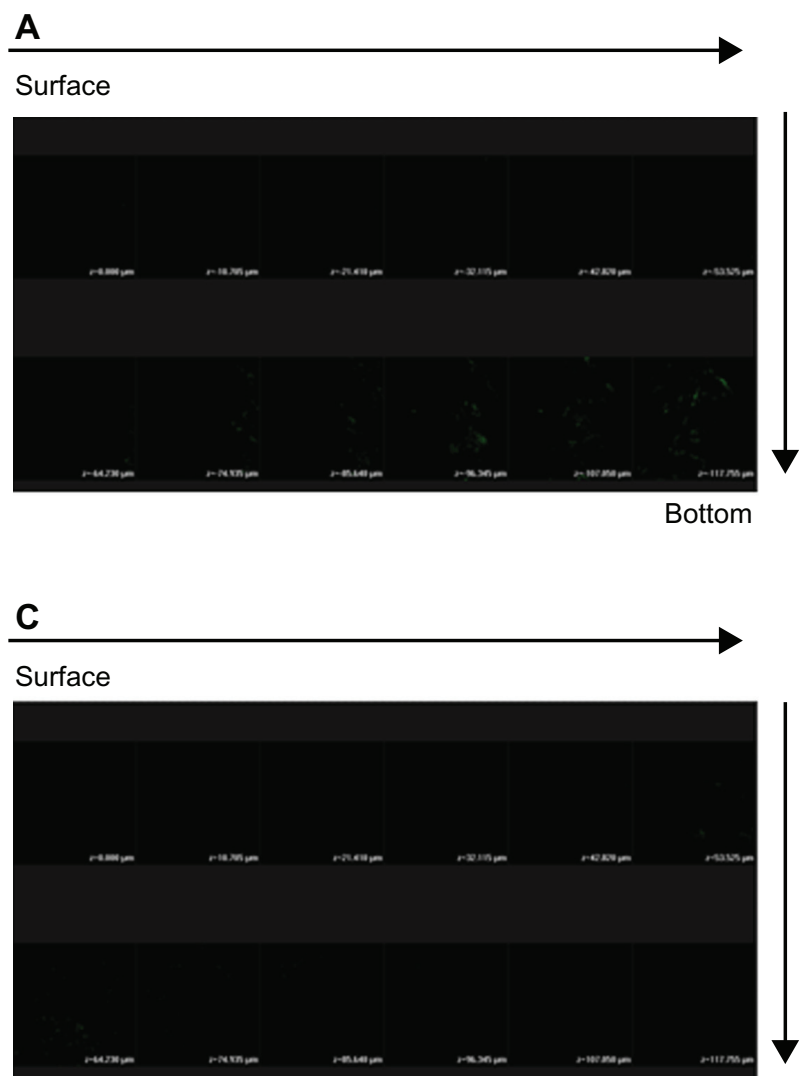

Bottom

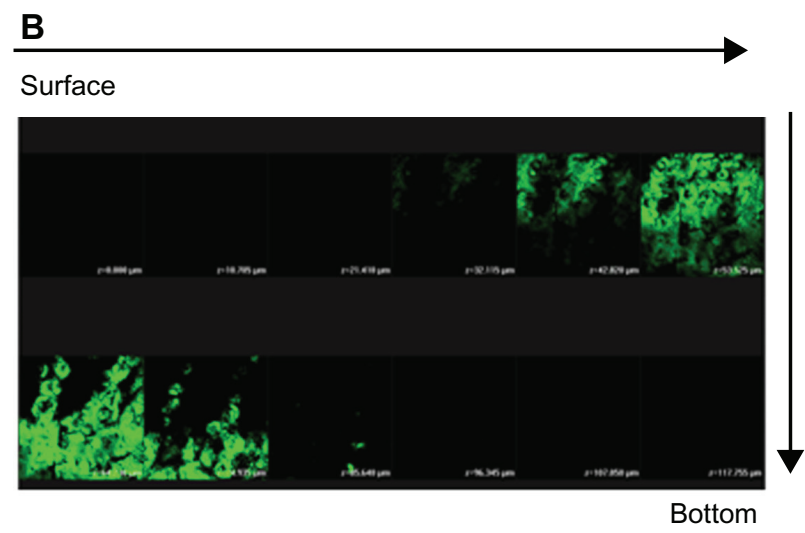

$\underline{\mathbf{D}}$

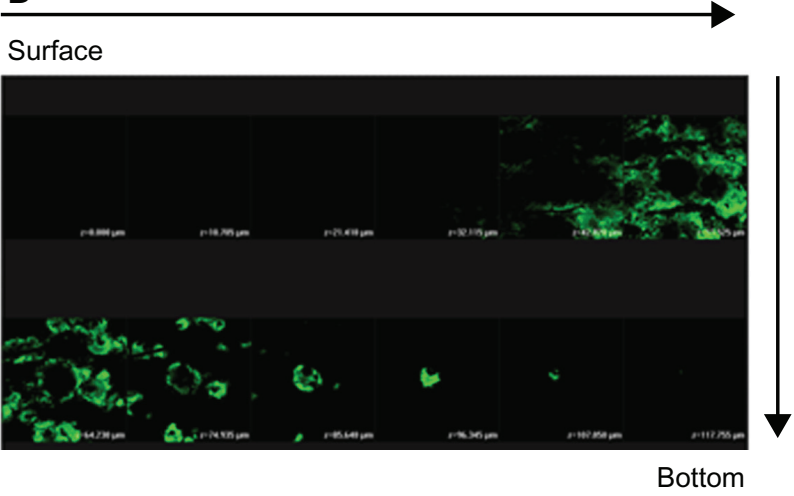

Figure 7 Confocal laser scanning microscopic (CLSM) micrographs of nude mouse skin after the in vivo topical administration of fluorescein isothiocyanate (FITC) via the skin for $2 \mathrm{~h}$ from 30\% ethanol (control) without sulforhodamine B (SRB) loading (A) NLC-5P without SRB loading (B) 30\% ethanol (control) with SRB loading (C) NLC-5P with SRB loading (D).

Notes: The skin specimen was viewed by CLSM at $\sim 10-\mu \mathrm{m}$ increments through the Z-axis from skin surface. The arrows indicate the skin fragments from surface to bottom.

might interact with-OH groups of calcipotriol. Many nitrogen molecules in methotrexate's structure can also donate lone pairs to attach to $-\mathrm{OH}$ of calcipotriol. This indicates that interactions between these two drugs might have played an important role in decreasing calcipotriol permeation. In the initial process of calcipotriol release, the drug undergoes a partitioning from the lipid phase to the aqueous phase. Then calcipotriol is released into the outer phase for subsequent partitioning into the SC. A large amount of methotrexate would interact with calcipotriol in the aqueous phase. This phenomenon may impede further calcipotriol permeation via skin or a cellulose membrane. The in vivo CLSM results did not show a reduction, since there was no interaction between SRB and FITC. However, this effect would negligibly influence methotrexate permeation, because only a small portion of methotrexate molecules in the aqueous phase would form a complex. Further study is needed to elucidate the detailed mechanisms of this possible interaction.

Methotrexate is hydrosoluble, and thus has higher affinity for the aqueous phase. ${ }^{36}$ Since this is the external phase in
NLCs, a greater amount of methotrexate is solubilized there. Because the diffusion of methotrexate is favored when it is incorporated into aqueous vehicles, we observed the effective methotrexate release from NLCs across a cellulose membrane. This also indicates that the lipid particles themselves did not significantly influence methotrexate release. The flux value of methotrexate was lower compared to the release rate of calcipotriol from all vehicles. This was due to a critical barrier function of the SC for the hydrophilic methotrexate. ${ }^{24,36}$ Both in vitro and in vivo permeation results revealed the enhanced delivery of methotrexate by NLC systems, with NLC-3P showing the greatest ability. The lipid particles must have some effects on the skin to induce this enhancement.

Similar to the case of calcipotriol, lipid particles form an adhesive layer occluding the skin surface. The hydration of the SC increases by reducing keratinocyte packing, and widening of the intercellular bilayers can facilitate drug penetration into deeper strata. This effect might be more pronounced for methotrexate than calcipotriol since adhesion increases with decreasing particle size. ${ }^{31,37}$ The penetration 


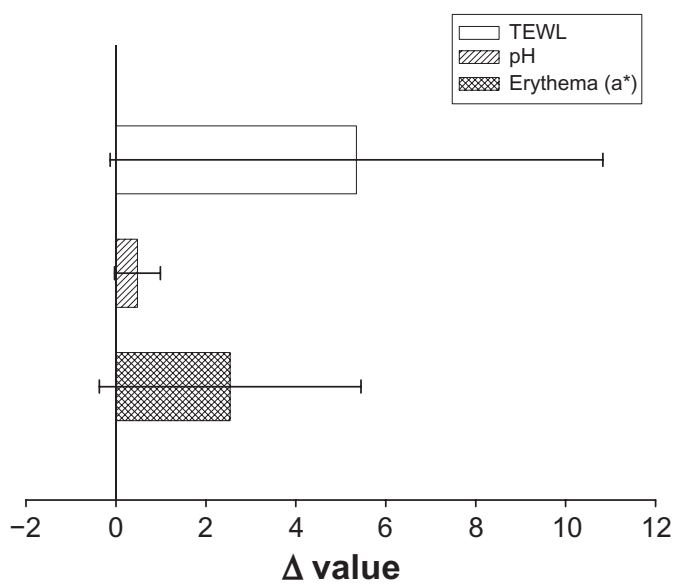

Figure 8 In vivo skin irritation examination determined by transepidermal water loss (TEWL), $\mathrm{pH}$ value, and erythema ( $\left.\mathrm{a}^{*}\right)$ after a 24-hour application of topically applied NLC-5P.

Notes: The $\Delta$ value indicates the value of the treated site minus the value of an adjacent untreated site. All data are presented as the mean of 6 experiments \pm SD.

of methotrexate from NLCs might not only be explained by an occlusive effect. Previous research ${ }^{35}$ suggested that the component emulsifiers in oil-in-water emulsions can reduce the functional barrier of the SC. Saturated and unsaturated fatty acids are frequently used as the lipid phase to enhance penetration. ${ }^{38}$ Precirol and Myverol in NLCs may act as penetration enhancers for methotrexate delivery. Clinical results with local methotrexate have been disappointing. ${ }^{39}$ A major problem is that the drug is hydrophilic, has a high molecular weight (455 Da), and is mostly in the dissociated form at a physiological $\mathrm{pH} .{ }^{2}$ NLCs can enhance the topical delivery of this drug to resolve this issue. A detailed investigation of the enhancing effect on drug permeation was not the main effort of this study. The basic mechanism should be elucidated in future studies.

Psoriasis is a disorder triggered when activated immunocytes infiltrate the skin, subsequently inducing prominent epidermal thickening. We used a tape-stripping technique to create hyperproliferative skin. It was confirmed that this method can induce psoriasis-like skin characterized by epidermal hyperplasia. It increased the epidermal thickness by 1.5 -fold. ${ }^{17}$ Calcipotriol flux showed no significant difference in hyperproliferative skin compared to normal skin. Although a longer pathway through which the drug had to pass was created by the psoriasis-like skin, a greater increase in methotrexate permeation via this skin was observed, especially for the NLC formulations. This may have been due to disruption of the permeability barrier in the hyperproliferative skin. ${ }^{14}$ Hence the NLC system can exert greater potency in treating psoriasis in disordered skin.
TEWL is used to assess the degree of SC disruption..$^{40}$ The $\mathrm{a}^{*}$-coordinate of colorimetry (which indicates erythema) has been demonstrated to correlate well with inflammatory interactions of the skin, especially in viable skin. NLCs showed no or only negligible increases in TEWL and a*. This suggests good tolerance of NLCs by the skin in this preliminary safety test. According to the hypothesis of enhancing drug permeation by NLCs, the nanoparticles as penetration enhancers may produce $\mathrm{SC}$ perturbations. The TEWL results indicated limited SC disruption by NLCs. It has been shown that the complete removal of lipids from the SC leads to a 100 -fold increase in water permeability. ${ }^{41}$

\section{Conclusions}

Psoriasis often reoccurs and is rarely cured, and hence patients should receive therapy periodically over many years. Thus, the development of vehicles for delivering multiple drugs to efficiently treat psoriasis is urgently needed. NLCs incorporating calcipotriol and methotrexate were developed and evaluated to achieve this aim. The present work confirmed that NLC systems are a promising carrier for the topical delivery of antipsoriatic drugs as revealed by enhanced skin permeation, negligible skin irritation, and the compatibility of the two drugs. A good correlation between in vitro and in vivo results was obtained. The skin permeation of methotrexate can be potentiated by using hyperproliferative skin as the penetration barrier. This is important for NLC administration on psoriatic skin. Most research related to topically applied SLNs or NLCs has demonstrated enhanced permeation of the drugs in lipid cores. We have proven that the drug in the aqueous phase also exhibits enhanced delivery with NLCs.

\section{Disclosures}

The authors declare no conflicts of interest.

\section{References}

1. Lowes MA, Bowcock AM, Krueger JG. Pathogenesis and therapy of psoriasis. Nature. 2007;445:866-872.

2. Su YH, Fang JY. Drug delivery and formulations for the topical treatment of psoriasis. Expert Opin Drug Deliv. 2008;5:235-249.

3. Vakirlis E, Kastanis A, Ioannides D. Calcipotriol/betamethasone dipropionate in the treatment of psoriasis vulgaris. Ther Clin Risk Manage. 2008;4:141-148.

4. Witman PM. Topical therapies for localized psoriasis. Mayo Clin Proc. 2001;76:943-949.

5. van der Kerkhof PCM, Cambazard F, Hutchinson PE, et al. The effect of addition of calcipotriol ointment $(50 \mu \mathrm{g} / \mathrm{g})$ to acitretin therapy in psoriasis. Br J Dermatol. 1998;138:84-89.

6. Ben-Shabat S, Benisty R, Wormser U, Sintov AC. Vitamin $\mathrm{D}_{3}$-based conjugates for topical treatment of psoriasis: synthesis, antiproliferative activity, and cutaneous penetration studies. Pharm Res. $2005 ; 22: 50-57$. 
7. Bowman PH, Maloney JE, Koo JY. Combination of calcipotriene $\left(\right.$ Dovonex $^{\circledR}$ ) ointment and tazarotene $\left(\right.$ Tazorac $^{\circledR}$ ) gel versus clobetasol ointment in the treatment of plaque psoriasis: a pilot study. $J$ Am Acad Dermatol. 2002;46:907-913.

8. Fogh K, Kragballe K. New vitamin D analogs in psoriasis. Curr Drug Target Inflamm Allergy. 2004;3:199-204.

9. Adachi Y, Uchida N, Matsuo T, Horio T. Clinical effect of vitamin $\mathrm{D}_{3}$ analogues is not inactivated by subsequent UV exposure. Photodermatol Photoimmunol Phatomed. 2008;24:16-18.

10. Pople PV, Singh KK. Development and evaluation of topical formulation containing solid lipid nanoparticles of vitamin A. AAPS Pharm Sci Tech. 2006;7:E1-E7.

11. Mandawgade SD, Patravale VB. Development of SLNs from natural lipids: application to topical delivery of tretinoin. Int J Pharm. 2008;363:132-138.

12. Müller RH, Petersen RD, Hommoss A, Pardeike J. Nanostructured lipid carriers (NLC) in cosmetic dermal products. Adv Drug Deliv Rev. 2007;59:522-530.

13. de Jong EMGJ, Mørk NJ, Seijger MMB, et al. The combination of calcipotriol and methotrexate compared with methotrexate and vehicle in psoriasis: results of a multicentre placebo-controlled randomized trial. Br J Dermatol. 2003;148:318-325.

14. Demerjian M, Mao MQ, Choi EH, et al. Topical treatment with thiazolidinediones, activators of peroxisome proliferators-activated receptor- $\gamma$, normalizes epidermal homeostasis in a murine hyperproliferative disease model. Exp Dermatol. 2006;15:154-160.

15. Ilic L, Gowrishankar TR, Vaughan TE, Herndon TO, Weaver JC. Spatially constrained skin electroporation with sodium thiosulfate and urea creates transdermal microconduits. $J$ Control Release. 1999;61:185-202.

16. Jenning V, Thünemann AF, Gohla SH. Characterization of a novel solid lipid nanoparticle carrier system based on binary mixtures of liquid and solid lipids. Int J Pharm. 2000;199:167-177.

17. Fang JY, Fang CL, Liu CH, Su YH. Lipid nanoparticles as vehicles for topical psoralen delivery: solid lipid nanoparticles (SLN) versus nanostructured lipid carriers (NLC). Eur J Pharm Biopharm. 2008;70:633-640.

18. Puglia C, Blasi P, Rizza L, et al. Lipid nanoparticles for prolonged topical delivery: an in vitro and in vivo investigation. Int $J$ Pharm. 2008;357:295-304

19. Wissing SA, Müller RH. Solid lipid nanoparticles as carrier for sunscreens: in vitro release and in vivo skin penetration. $J$ Control Release. 2002;81:225-233.

20. Weyenberg W, Filev P, Van den Plas D, et al. Cytotoxicity of submicron emulsions and solid lipid nanoparticles for dermal application. Int J Pharm. 2007;337:291-298.

21. Jores K, Haberland A, Wartewig S, Mäder K, Mehnert W. Solid lipid nanoparticles (SLN) and oil-loaded SLN studied by spectrofluorometry and Raman spectroscopy. Pharm Res. 2005;22:1887-1897.

22. Lombardi Borgia S, Regehly M, Sivaramakrishnan R, et al. Lipid nanoparticles for skin penetration enhancement - correlation to drug localization within the particle matrix as determined by fluorescence and parelectric spectroscopy. J Control Release. 2005;110:151-163.

23. Baroli B, López-Quintela MA, Delgado-Charro MB, Fadda AM, BlancoMéndez J. Microemulsions for topical delivery of 8-methoxsalen. J Control Release. 2000;69:209-218.
24. Dubey V, Mishra D, Dutta T, Nahar M, Saraf DK, Jain NK. Dermal and transdermal delivery of an anti-psoriatic agent via ethanolic liposomes. J Control Release. 2007;123:148-154.

25. Venkateswarlu V, Manjunath K. Preparation, characterization and in vitro release kinetics of clozapine solid lipid nanoparticles. $J$ Control Release. 2004;95:627-638.

26. Müller RH, Mäder K, Gohla S. Solid lipid nanoparticles (SLN) for controlled drug delivery - a review of the state of the art. Eur J Pharm Biopharm. 2000;50:161-177.

27. Jenning V, Schäfer-Korting M, Gohla S. Vitamin A-loaded solid lipid nanoparticles for topical use: drug release properties. J Control Release. 2000;66:115-126.

28. Bunjes H, Drechsler M, Koch MHJ, Westesen K. Incorporation of the model drug ubidecarenone into solid lipid nanoparticles. Pharm Res. 2001;18:287-293.

29. Hu FQ, Jiang SP, Du YZ, Yuan H, Ye YQ, Zeng S. Preparation and characteristics of monostearin nanostructured lipid carriers. Int J Pharm. 2006;314:83-89.

30. Müller RH, Radtke M, Wissing SA. Solid lipid nanoparticles (SLN) and nanostructured lipid carriers (NLC) in cosmetic and dermatological preparations. Adv Drug Deliv Rev. 2002;54:S131-S155.

31. Chen H, Chang X, Du D, et al. Podophyllotoxin-loaded solid lipid nanoparticles for epidermal targeting. $J$ Control Release. 2006;110:296-306.

32. Küchler S, Radowski MR, Blaschke T, et al. Nanoparticles for skin penetration enhancement - a comparison of a dendritic core-multishellnanotransporter and solid lipid nanoparticles. Eur J Pharm Biopharm. 2009; $71: 243-250$

33. Souto EB, Almeida AJ, Müller RH. Lipid nanoparticles (SLN ${ }^{\circledR}$, $\mathrm{NLC}^{\circledR}$ ) for cutaneous drug delivery: structure, protection and skin effects. J Biomed Nanotechnol. 2007;3:317-331.

34. Bourke JF, Berth-Jones J, Hutchinson PE. Occlusion enhances the efficacy of topical calcipotriol in the treatment of psoriasis vulgaris. Clin Exp Dermatol. 1993;18:504-506.

35. Nielsen PG. Calcipotriol or clobetasol propionate occluded with a hydrocolloid dressing for treatment of nummular psoriasis. Acta Derm Venereol. 1993;73:394.

36. Alvarez-Figueroa MJ, Blanco-Méndez J. Transdermal delivery of methotrexate: iontophoretic delivery from hydrogels and passive delivery from microemulsions. Int J Pharm. 2001;215:57-65.

37. Schäfer-Korting M, Mehnert W, Korting H. Lipid nanoparticles for improved topical application of drugs for skin diseases. Adv Drug Deliv Rev. 2007;59:427-443.

38. Kogan A, Garti N. Microemulsions as transdermal drug delivery vehicles. Adv Colloid Interf Sci. 2006;123-126:369-385.

39. Wong TW, Chen CH, Huang CC, Lin CD, Hui SW. Painless electroporation with a new needle-free microelectrode array to enhance transdermal drug delivery. J Control Release. 2006;110:557-565.

40. Zhao K, Singh J. In vitro percutaneous absorption enhancement of propranolol hydrochloride through porcine epidermis by terpenes/ ethanol. J Control Release. 1999;62:359-366.

41. Serup J, Agner T. Colorimetric quantification of erythema - a comparison of two colorimeters (Lange Micro Color and Minolta Chroma Meter CR-200) with a clinical scoring scheme and laser-Doppler flowmetry. Clin Exp Dermatol. 1990;15:267-272.
International Journal of Nanomedicine

\section{Publish your work in this journal}

The International Journal of Nanomedicine is an international, peerreviewed journal focusing on the application of nanotechnology in diagnostics, therapeutics, and drug delivery systems throughout the biomedical field. This journal is indexed on PubMed Central, MedLine, CAS, SciSearch ${ }^{\circledR}$, Current Contents ${ }^{\circledR} /$ Clinical Medicine,
Dovepress

Journal Citation Reports/Science Edition, EMBase, Scopus and the Elsevier Bibliographic databases. The manuscript management system is completely online and includes a very quick and fair peer-review system, which is all easy to use. Visit http://www.dovepress.com/ testimonials.php to read real quotes from published authors. 